\title{
A METHOD OF CONSTRUCTING PROGRAMMED MOTIONS OF A MECHANICAL SYSTEM
}

\author{
DO SANH \\ Hanoi University of Technology
}

\begin{abstract}
In the paper the method for constructing programmed motion is represented. The requirements for the programmed motion are treated as ideal constraints in analytical mechanics. The programmed motions expressed in lagrange coordinates and in quasi coordinates are investigated.

By applying the form of equations of motion of a constrained mechanical system [7], a schema for calculating programmed motions has been established. By this schema the errors of realizing programmed motions have been reduced and controlled.
\end{abstract}

For illustration of the method some examples have been investigated.

\section{$\S 1$. Introduction}

In the theory of controlled motion, there exist some requirements, that restrict the motion of the system under consideration.

In other words, the process occurring in this system must satisfy some preset requirements. The conditions, that a process with specified properties the system must realize, are called the programmed and the motion of the system corresponding to this process is named the programmed motion.

The problem of programmed motion has been investigated by many authors $[2,8,9 \ldots]$.

In $[4,5]$ for constructing programmed motion the author has used the principle of compatibility, which gives an unique point of view for investigating this problem. By this it is possible not only to calculate the needful controlling forces for realizing the given programmed motion, but to find out their construction - this fact serves for qualitative investigations of dynamical processes, for example, stable or optimal investigations, etc,...

However, in the mentioned method it can meet errors in realizing programmed motions caused by calculating for example, the inverse of the matrix of inertia. This results the deviation from the required programme and sequently the pro- 
gramme is violated.

In this work a method, that allows to overcome these difficulties, is represented.

\section{Construction of a programmed motion given in Lagrangian coordinates}

Let us consider a holonomic mechanical system. Denote the generalized coordinates by $q_{i}(i=\overline{1, n})$ and the generalized forces corresponding to them by $Q_{i}\left(t, q_{j}, \dot{q}_{j}\right)$. The $n \times 1$ matrix of generalized forces is designated by $\mathbf{Q}$. Kinetic energy of the system is of the form

$$
T=\frac{1}{2} \dot{\mathbf{q}}^{T} \mathbf{A} \dot{\mathrm{q}}
$$

where $\mathbf{A}$ is a quadratic symmetric and inversible matrix of order $n$. The elements of this matrix depend on generalized coordinates, but $\dot{q}-$ the $(n \times 1)$ matrix of generalized velocities. The letter $T$ located on the right corner designated a transposed matrix.

In brief, a mechanical system with the kinetic energy $T$ and the generalized forces matrix $\mathbf{Q}$ is called the nature system.

As known [3], the motion of a nature system is described by equations

$$
\mathbf{A} \ddot{\mathrm{q}}-\mathbf{G}=\mathbf{Q}
$$

where $\mathbf{G}$ is an $(n \times 1)$ matrix, the elements of which are only drawn from the matrix of inertia.

The problem of constructing the programmed motion is stated as follows:

Design a mechanical system in such a way so that the programmed motion of the form

$$
g_{\beta}(t, \mathbf{q}, \dot{\mathbf{q}})=0 \quad \beta=\overline{\mathbf{1 , s}}
$$

is one of the possible motions of this system. In other words, the trajectories of the designed system must locate on the manifold (2.3). We assume that the equations (2.3) are compatible and independent in a certain given domain $\Omega$ of the phase space for all $t \geq t_{0}$.

It is clear, that the subject of the present consideration associated with the problem of synthesis of a system performing a prescribed motion. 
The programme is called to be complete when $s=n$ and uncomplete when $s<n$.

First, we shall consider the case of performing the programmed motion (2.3) with the help of additionat controlling forces. Such a system is named a open one.

Equations of motion of the system realizing the programme (2.3) can be written as follows:

$$
\mathbf{A} \ddot{\mathbf{q}}-\mathbf{G}=\mathbf{Q}+\mathbf{u}
$$

where $\mathbf{u}$ is the $(n \times 1)$ matrix of the controlling forces required.

Of course, the motion of the nature system (2.2) are not in general, identical with the programme (2.3). The problem is stated now as follows:

Find the controlling forces $u$ so that the system (2.4) realizes the programmed motion (2.3).

Here we shall confine overselves to a consideration of the case of incomplete programme, i.e., $s<n$. In the case there is no restrictions putting on the controlling forces it is possible to treat (2.3) as the equations of ideal constraints in analytical mechanics, but the controlling forces - the reaction forces of these constraints. This is quite agreable with the postulate of constraints in analytical mechanics: "It is possible to regard a constrained system as a free one (a freed system) if the constraints should be substituted by their reaction forces". For raison of compatibility, the initial conditions must satisfy the programme, i.e.

$$
\left.g_{\beta}\left(t_{0}, \mathbf{q}\left(t_{0}\right), \dot{\mathbf{q}}\right)\left(t_{0}\right)\right)=0
$$

We also assume that all functions that are encountered in this work are continuous together with their derivatives in the considered domain $\Omega$, where the rank of Jacobi mairix

$$
\left\|\frac{\partial g_{\beta}}{\partial \dot{q}_{i}}\right\|
$$

is equal to $s$ for all $t \geq t_{0}$ and $\mathbf{q}, \dot{\mathbf{q}} \ni \Omega$. In addition we suppose that algebraic equations are compatible and differential equations satisfy conditions of existence and uniqueness of solution.

Because the programme (2.3) is treated as a set of ideal constraints and $\mathbf{u}$ is the reaction forces matrix we have then $[3,6,7]$

$$
\mathbf{D} \mathbf{u}=\mathbf{0}
$$

where $\mathbf{D}$ is an $(n-s) \times n$ matrix the elements of this matrix are coefficients in the expression of accelerations written in terms of independent accelerations. 
From (2.4) and (2.7) we draw:

$$
\mathbf{D}^{*} \ddot{\mathbf{q}}=\mathbf{Q}^{*}
$$

where the matrices $\mathbf{D}^{*}$ and $\mathbf{Q}^{*}$ are of the form

$$
\begin{gathered}
\mathbf{D}^{*}=\mathbf{D} \mathbf{A} \\
\mathbf{Q}^{*}=\mathbf{D} \mathbf{G}+\mathbf{D} \mathbf{Q} .
\end{gathered}
$$

The matrix $\mathbf{D}^{*}$ has $(n-s) \times n$ dimension, but $\mathbf{Q}^{*}-$ the $(n \times 1)$ dimension.

In such a way we obtain $n$ equations (2.8) and (2.3), which describe the motion of the designed system.

It is noticed that equations (2.8) and (2.3) are of a set of algebraic - differential equations. Let

$$
\mathbf{q}=\mathbf{q}(t), \quad \dot{\mathbf{q}}=\dot{\mathbf{q}}(t), \quad \ddot{\mathbf{q}}=\ddot{\mathbf{q}}(t)
$$

be the solution of the equations (2.8) (2.3) with the initial conditions, which satisfy (2.5)

The controlling forces then are calculated by (2.4), that are

$$
\mathbf{u}(t)=\mathbf{A}(\mathbf{q}(t)) \ddot{\mathbf{q}}-\mathbf{Q}(t, \mathbf{q}(t), \dot{\mathbf{q}}(t))-\mathbf{G}(t, \mathbf{q}(t), \dot{\mathbf{q}}(t))
$$

By of acting of these additional forces the system will realize the programme (2.3).

Differing from the method represented in $[4,5]$ in the above given algorithm it is unnecessary to calculate the inverse of the matrix of inertia. This reduces calculating errors of realizing the programmed motion.

It is important that the equations of required programme are directly included in a closed set of equations described the motion of the system under consideration. Therefore, the errors of the required programme just are ones of computed algorithm. This allows to control the deviation of the programme in the process of realizing it.

Example 2.1. Let us consider the motion of a particle of mass $m$ in Newtonian field of attraction force $[2,5]$.

Determine the controlling forces so that the module of the velocity vector of the particle is constant. Let us choose the generalized coordinates to be spherical coordinates $\theta, \varphi, r$. 
The cartesian coordinates of the particle are of the form $x=r \cos \theta \cos \varphi$, $y=r \cos \theta \sin \varphi, z=r \sin \theta$. It is easy to calculate the module of the velocity vector of the particle:

$$
v=\sqrt{\dot{x}^{2}+\dot{y}^{2}+\dot{z}^{2}}=\sqrt{\dot{r}^{2}+r^{2} \cos ^{2} \theta \dot{\varphi}^{2}+r^{2} \dot{\theta}^{2}} .
$$

Kinetic energy of the particle is now

$$
T=\frac{1}{2} m\left(\dot{r}^{2}+r^{2} \cos ^{2} \theta \dot{\varphi}^{2}+r^{2} \dot{\theta}^{2}\right) .
$$

The $(3 \times 3)$ matrix of inertia has the form

$$
\mathbf{A}=\left[\begin{array}{ccc}
r^{2} & 0 & 0 \\
0 & r^{2} \cos ^{2} \theta & 0 \\
0 & 0 & 1
\end{array}\right]
$$

but the potential function will be

$$
\Pi=-\frac{\gamma m M}{r^{2}}=-\frac{\mu m}{r^{2}}
$$

where: $\mu=\gamma M$, but $\gamma$ is the gravitation constant, $M$-the mass of gravitational centre. The $3 \times 1$ matrix $Q$ takes the form

$$
\mathrm{Q}^{T}=\left\|\begin{array}{lll}
0 & 0 & -\frac{\mu m}{r} \|
\end{array}\right\|
$$

The condition of constant module of velocity vector of the particle complies the programmed constraint of the form

$$
g(\theta, \varphi, r, \dot{\theta}, \dot{\varphi}, \dot{r}) \equiv r^{2} \dot{\theta}^{2}+r^{2} \cos ^{2} \dot{\varphi}^{2}+\dot{r}^{2}-C=0
$$

which is treated as an ideal constraint. The $(2 \times 3)$ matrix $D$ in the formula (2.7) has the form

$$
\mathbf{D}=\left\|\begin{array}{ccc}
1 & 0 & -r^{2} \dot{\theta} / \dot{r} \\
0 & 1 & -r^{2} \cos ^{2} \theta \dot{\varphi} / \dot{r}
\end{array}\right\|
$$

The $(3 \times 1)$ matrix $G$ will be now

$$
\mathbf{G}=\left\|\begin{array}{l}
-m\left(2 r \dot{\theta} \dot{r}+r^{2} \cos \theta \sin \theta \dot{\varphi}^{2}\right) \\
-2 m\left(r \cos ^{2} \theta \dot{r} \dot{\varphi}+r^{2} \cos \theta \sin \theta \dot{\theta} \dot{\varphi}\right) \\
m\left(r \cos ^{2} \theta \dot{\varphi}^{2}+r \dot{\theta}^{2}\right)
\end{array}\right\| .
$$


In accordance with the formulas (2.9) and (2.10) the $(2 \times 3)$ matrix $\mathbf{D}^{*}$ and the $(2 \times 1)$ matrix $Q^{*}$ take the forms respectively

$$
\begin{gathered}
\mathbf{D}^{*}=\left\|\begin{array}{ccc}
m r^{2} & 0 & -m r^{2} \dot{\theta} / \dot{r} \\
0 & m r^{2} \cos ^{2} \theta & -m r^{2} \cos ^{2} \theta \dot{\varphi} / \dot{r}
\end{array}\right\| \\
\mathbf{Q}^{*}=\left\|\begin{array}{c}
-m\left(2 r \dot{r} \dot{\theta}+r^{2} \cos \theta \sin \theta \dot{\varphi}^{2}\right)-r^{2} m\left[\left(r \cos ^{2} \theta \dot{\varphi}^{2}+r \dot{\theta}^{2}\right)-\frac{\mu}{r}\right] \frac{\dot{\theta}}{\dot{r}} \\
-2 m\left(r \cos ^{2} \theta \dot{r} \dot{\varphi}+r^{2} \cos \theta \sin \theta \dot{\theta} \dot{\varphi}\right)-m r^{2} \cos ^{2} \theta\left[\left(r \cos ^{2} \theta \dot{\varphi}^{2}+r \dot{\theta}^{2}\right)-\frac{\mu}{r}\right] \frac{\dot{\varphi}}{\dot{r}}
\end{array}\right\|
\end{gathered}
$$

Equations (2.8) and (2.3) described the motion of the system under consideration take the form now

$$
\begin{gathered}
r \dot{r} \ddot{\theta}-r \dot{\theta} \ddot{r}=-\left(2 \dot{r}^{2} \dot{\theta}+r \cos \theta \sin \theta \dot{r} \dot{\varphi}^{2}\right)-\left[r^{2}\left(\cos ^{2} \theta \dot{\varphi}^{2}+\dot{\theta}^{2}\right)-\mu r\right] \dot{\theta} \\
r \dot{r} \cos ^{2} \theta \ddot{\varphi}-r \cos ^{2} \theta \dot{\varphi} \ddot{r}=-2\left(\cos ^{2} \theta \dot{r}^{2} \dot{\varphi}+r \cos \theta \sin \theta \dot{\theta} \dot{\varphi} \dot{r}\right) \\
-\left[r^{2} \cos ^{2} \theta\left(\cos ^{2} \theta \dot{\varphi}^{2}+\dot{\theta}^{2}\right)-\mu r\right] \dot{\varphi}, \\
r \dot{\theta}^{2}+r^{2} \cos ^{2} \theta \dot{\varphi}^{2}+\dot{r}^{2}-C=0 .
\end{gathered}
$$

In [2] this problem has been investigated by means of the Lagrange's equations with multipliers. It is easy to see that by eliminating multipliers we will obtain the above established equations.

Let the solution of these equations with the given conditions be of the form

$$
\theta=\theta(t, C), \quad \varphi=\varphi(t, C), \quad r=r(t, C)
$$

and substituting them into (2.12) we get the required controlling forces, that are

$$
u_{\theta}=u_{\theta}(t, C), \quad u_{\varphi}=u_{\varphi}(t, C), \quad u_{r}=u_{r}(t, C)
$$

The programmed motion (2.18) can be realized, of course, by one controlling parameter $C$.

\section{§3. Construction of a programmed motion given in quasi coordinates}

First, it is noticed that the Appell's equations written in the Lagrange's coordinates take the same form as (2.2). In other words, the Gauss' function is written as follows: $[5]$

$$
U=\frac{1}{2} \ddot{\mathbf{q}}^{T} \mathbf{A} \ddot{\mathbf{q}}-\mathbf{G} \ddot{\mathbf{q}}+\ldots
$$


where the now written terms don't contain accelerations, but $\mathbf{A}$ and $\mathbf{G}$ have the same meaning as in (2.2).

Suppose that the quasi velocities are of the form

$$
\omega_{s}=\sum_{r=1}^{n} C_{s r} \dot{q}_{r}, \quad s=\overline{1, n}
$$

which in the matrix form is written as follows

$$
\boldsymbol{\omega}=\mathbf{C} \dot{\mathbf{q}}
$$

where $\boldsymbol{\omega}$ is an $(n \times 1)$ matrix, but $\mathbf{C}$ - a inversible quadratic matrix of order $n$. Equations of motion of the nature system (2.2) in quasi coordinates take the form

$$
\mathbf{A}_{0} \dot{\boldsymbol{\omega}}=\mathbf{G}_{0}+\mathbf{Q}_{0}-\mathbf{A} \mathbf{C}^{*} \boldsymbol{\omega}
$$

where $\boldsymbol{C}^{*}$ is the inverse of $\mathbf{C}$, but

$$
\begin{gathered}
\mathbf{A}_{0}=\mathbf{A C}^{*} \\
\cdot \mathbf{G}_{0}=\mathbf{G}(\mathbf{q}, \dot{\mathbf{q}}(\boldsymbol{\omega})), \quad \mathbf{Q}_{0}=\mathbf{Q}(t, \mathbf{q}, \dot{\mathbf{q}}(\boldsymbol{\omega})) .
\end{gathered}
$$

Suppose that the programme, that must be realized, is of the form

$$
g_{\beta}(t, \mathbf{q}, \boldsymbol{\omega})=0, \quad \beta=\overline{1, s} .
$$

Let us consider the case of open controlled systems. As above, it can act additional controlling forces on the system so that under action of these forces the system will realize the programmed motion (3.7).

Equations of motion of the system are written in the form then

$$
\mathbf{A}_{0} \dot{\boldsymbol{\omega}}=\mathbf{G}_{0}+\mathbf{Q}_{0}-\mathbf{A} \dot{\mathbf{C}}^{*} \boldsymbol{\omega}+\mathbf{u}(\mathbf{q}, \boldsymbol{\omega}, t)
$$

where $\mathbf{u}$ is the $(n \times 1)$ matrix of controlling forces.

In the case there is no any restrictions putting on the controlling forces it is possible to treat the programme (3.7) as ideal constraints in analytical mechanics.

The condition of ideality of the constraints (3.7) take the following form

$$
\mathbf{D}_{0} \mathbf{u}=\mathbf{0}
$$

where $\mathbf{D}_{0}$ is the $(n-s) \times n$ matrix, which is constructed in the same way of the matrix $\mathbf{D}$ in (2.7). 
In accordance with (3.8) and (3.9) we obtain

$$
\mathbf{D}^{*} \dot{\boldsymbol{\omega}}=\mathbf{\Omega}
$$

where $\mathbf{D}^{*}$ is the $(n-s) \times n$ matrix, which has the form

$$
\mathbf{D}^{*}=\mathbf{D}_{0} \mathbf{A}_{0}
$$

but $\boldsymbol{\Omega}$ is the $(n \times 1)$ matrix, which is of the form

$$
\mathbf{\Omega}=\mathbf{D}_{0} \mathbf{G}_{0}+\mathbf{D}_{0} \mathbf{Q}_{0}-\mathbf{D}_{0} \mathbf{A} \dot{\mathbf{C}}^{*} \mathbf{\Omega}
$$

Equations (3.10) and (3.7) describe the motion of the system under consideration.

Let $q(t), \omega(t)$ and $\dot{\omega}(t)$ is the solution of these equations with the given initial conditions. The controlling forces required are calculated by the formula

$$
\begin{aligned}
\mathbf{u}(t)= & \mathbf{A}_{0}(\mathbf{q}(t), \boldsymbol{\omega}(t)) \dot{\boldsymbol{\omega}}(t)-\mathbf{G}_{0}(\mathbf{q}(t), \boldsymbol{\omega}(t)) \\
& -\mathbf{Q}_{0}(t, \mathbf{q}(t), \boldsymbol{\omega}(t))-\mathbf{C}^{*}(\mathbf{q}(t)) \boldsymbol{\omega}(t)
\end{aligned}
$$

Under action of these forces the system under consideration will realize the programmed motion (3.7).

Note - In the case when the equations of motion of the nature system are written in quasi coordinates, for example, in the Appell's equations by means of quasi coordinates it can directly apply these equations.

Example 3.1. Find the controlling forces imposed on a body in order that the motion is of generalized pression of the vector $\boldsymbol{\omega}$.

As known (see, for example $[3,4,5]$, the condition of generalized pression of the vector $\boldsymbol{\omega}$ is equivalent to the programmed constraint of the form.

$$
p \dot{q}-q \dot{p}+r\left(p^{2}+q^{2}\right)-\lambda\left(p^{2}+q^{2}\right)^{3 / 2}=0
$$

where $p, q, r$ are of the projections of the vector of the instantaneous angular velocity vector $\omega$ on the rectangular orthogonal axes associated with the body, but $\lambda$ is an arbitrary constant.

The Gauss function is of the form

$$
\begin{aligned}
U= & \frac{1}{2}\left(A \dot{p}^{2}+B \dot{q}^{2}+C \dot{r}^{2}\right)+2(C-B) q r \dot{p}+2(A-C) r p \dot{q} \\
& +2(B-A) p q \dot{r}-Q_{p} \dot{p}-Q_{q} \dot{q}-Q_{r} \dot{r}+\ldots
\end{aligned}
$$


where the nonwritten terms don't contain $\dot{p}, \dot{q}, \dot{r}$, but $A, B, C$ are the moments of inertia of the body about the principal axes of inertia.

Equations of motion of the nature system can be written as follows $[1,2]$

$$
\begin{aligned}
& A \dot{p}+(C-B) q r=Q_{p} \\
& B \dot{q}+(A-C) p r=Q_{p} \\
& C \dot{r}+(B-A) p q=Q_{r}
\end{aligned}
$$

where: $Q_{p}, Q_{q}, Q_{r}$ are the generalized forces corresponding to quasi coordinates

The relation to power gives us:

$$
p Q_{p}+q Q_{q}+r Q_{r} \equiv \dot{\varphi} Q_{\varphi}+\dot{\theta} Q_{\theta}+\dot{\psi} Q_{\psi}
$$

where: $\varphi, \theta, \psi$ are the Euler's angles, but $Q_{\varphi}, Q_{\vartheta}, Q_{\psi}$-the generalized forces respectively.

As known $[1,2]$ we have

$$
\begin{aligned}
& p=\dot{\psi} \sin \theta \sin \varphi+\dot{\theta} \cos \varphi \\
& q=\dot{\psi} \sin \theta \cos \varphi-\dot{\theta} \sin \varphi \\
& r=\dot{\psi} \cos \theta+\dot{\varphi}
\end{aligned}
$$

Substituting (3.17) into (3.16) we obtain:

$$
\begin{aligned}
& Q_{p}=-\operatorname{ctg} \theta Q_{\varphi}+\cos \varphi Q_{\theta}+\frac{\sin \varphi}{\sin \theta} Q_{\psi}, \\
& Q_{q}=-\operatorname{ctg} \theta \cos \varphi Q_{\varphi}-\sin \varphi Q_{\theta}+\frac{\cos \varphi}{\sin \theta} Q_{\psi}, \\
& Q_{r}=Q_{\varphi} .
\end{aligned}
$$

As above, equations of motion of the system, realizing the programme (3.14) can be written in the form:

$$
\begin{aligned}
& A \dot{p}+(C-B) q r=Q_{p}+u_{p} \\
& B \dot{q}+(A-C) r p=Q_{q}+u_{q} \\
& C \dot{r}+(B-A) p q=Q_{r}+u_{r}
\end{aligned}
$$

where: $u_{p}, u_{q}, u_{r}$ are the controlling forces in order that under action of which the system realizes the programme (3.14). 
The $(2 \times 3)$ matrix $\mathbf{D}_{0}$ in $(3.9)$ has the form now

$$
\mathbf{D}_{0}=\left\|\begin{array}{ccc}
1 & q / p & 0 \\
0 & 0 & 1
\end{array}\right\|
$$

Equations (3.10) take the form now

$$
\begin{aligned}
& p[A \dot{p}+(C-B) q r-Q p]+q\left[B \dot{q}+(A-C) r p-Q_{p}\right]=0 \\
& C \dot{r}+(B-A) p q=Q_{r} .
\end{aligned}
$$

The first of equations (3.21) can be written as follows:

$$
A p \dot{p}+B q \dot{q}+(A-B) p q r-p Q_{p}-q Q_{q}=0
$$

From (3.16) we have

$$
\begin{aligned}
p Q_{p}+q Q_{q} & =\dot{\varphi} Q_{\varphi}+\dot{\theta} Q_{\theta}+\dot{\psi} Q_{\psi}-r Q_{r} \\
& =\dot{\theta} Q_{\theta}+\dot{\psi}\left(Q_{\psi}-\cos \theta Q_{\varphi}\right)
\end{aligned}
$$

Equations of motion of the system, which must realize the programmed motion (3.7) are of the form now

$$
\begin{aligned}
& A p \dot{p}+B q \dot{q}+(A-B) p q r-\dot{\theta} Q_{\theta}+\dot{\psi}\left(\cos \theta Q_{\varphi}-Q_{\psi}\right)=0 \\
& C \dot{r}+(B-A) p q=Q_{\varphi} \\
& p \dot{q}-q \dot{p}+r\left(p^{2}+q^{2}\right)-\lambda\left(p^{2}+q^{2}\right)^{3 / 2}=0
\end{aligned}
$$

Let the solution of these equations with the given initial conditions be

$$
\begin{array}{ll}
p=p(t), \dot{p}=\dot{p}(t), \quad q=q(t), \dot{q}=\dot{q}(t), \\
r=r(t), \dot{r}=\dot{r}(t), \quad \psi=\psi(t), \varphi=\varphi(t), \quad \theta=\theta(t)
\end{array}
$$

and therefore:

$$
\begin{aligned}
& Q_{\varphi}=Q_{\varphi}(t), Q_{\theta}=Q_{\theta}(t), Q_{\psi}=Q_{\psi}(t), Q_{p}=Q_{p}(t) \\
& Q_{q}=Q_{q}(t), Q_{r}=Q_{\varphi}(t)
\end{aligned}
$$

From (3.19) we calculate the controlling forces for realizing the programme (3.14), that are:

$$
\begin{aligned}
& u_{p}(t)=A \dot{p}(t)+(C-B) q(t) r(t)-Q_{p}(t), \\
& u_{q}(t)=B \dot{q}(t)+(A-C) r(t) p(t)-Q_{q}(t) \\
& u_{r}(t)=C \dot{r}(t)+(B-A) p(t) q(t)-Q_{\varphi}(t)
\end{aligned}
$$


It is easy to see that the equations (3.24) can be leaded to ones established [3].

\section{$\S 4$. Conclusion}

In the work an algorithm for calculating a programmed motion has investigated. The requirements for a programmed motion are treated as ideal constraints in analytical mechanics.

The following results have been obtained

1) A schema for calculating programmed motion have been established. By represented schema the calculation of the inverse of the matrix of inertia has been avoided. This result has an important meaning for numerical methods, especially in the case of the systems of big dimension.

2) In represented schema, the equations of programmed motion include in the closed set of equations described the motion of the system under consideration. This allows to control the errors of realizing the given programmed motion.

This work is completed with financial support of the council for Natural Sciences of Vietnam

\section{REFERENCES}

1. Appell. P. Traite de mecanique rationnelle, Paris, tom II, Gauthier-Villars, 1953.

2. Dobronravov V. V. The bases of nonholonomic mechanics, "Vysha skola", Moscow, 1970 (in Russian).

3. Do Sanh. A form of equations of motion of a mechanical system, Journal of Mechanics, NCNST of Vietnam, № 31995.

4. Do Sanh. On the motion of a controlled mechanical system, Advances in Mechanics, Vol 7, № 2, Warsaw, 1984.

5. Do Sanh. On the motion of constrained mechanical system, The thesic of Science Dotor, Hanoi, 1984.

6. Do Sanh. A form of equations of motion of a constrained mechanical system, Proceedings of International Conference on Applied Dynamics, Hanoi 1995.

7. Do Sanh. A new form of equations of motion of a mechanical system, Journal of Mechanics, NCNST of Vietnam, T.XIX, № 3, 1997.

8. Galiullin A. S., et al. Construction Programmed motion systems, Nauka, Moscow, 1971 (in Russian). 
9. Galiullin A. S. Inverse problems of Dynamics Mir Publishers Moscow, 1984.

Received November 12, 1998

XÂY DỰNG CHUYỂN ĐộNG CHUONG TRÌNH CƯA HỆ CƠ HỌC

Trong bài báo trình bày một phương pháp xây dựng chuyển động chương trình của hệ cơ học. Các yêu cầu đối với chương trình được xem như là những liên kết lý tương trong cơ học giải tích. Đã khảo sát chương trình được biểu diễn trong tọa độ Lagrang và trong tựa tọa độ.

Để giải quyết bài toán đã áp dụng trực tiếp dạng phương trình chuyển động của cơ hệ được trình bày trong [7]. Đã đưa ra một số sơ đồ cho việc tính toán các chuyển động chương trình. Với sơ đồ này sai số trong việc tính toán chuyển động chương trình đã được giảm và được khống chế.

Đã khảo sát một số thí dụ để minh họa cho phương pháp đưa ra. 